\title{
Stance Detection: \\ Concepts, Approaches, Resources, and Outstanding Issues
}

\author{
Dilek Küçük \\ TÜBİTAK MRC Energy Institute \\ Electrical Power Technologies Department \\ Ankara, Turkey \\ dilek.kucuk@tubitak.gov.tr
}

\author{
Fazli Can \\ Bilkent University \\ Computer Engineering Department \\ Ankara, Turkey \\ canf@cs.bilkent.edu.tr
}

\begin{abstract}
Stance detection (also known as stance classification and stance prediction) is a problem related to social media analysis, natural language processing, and information retrieval, which aims to determine the position of a person from a piece of text they produce, towards a target (a concept, idea, event, etc.) either explicitly specified in the text, or implied only. The output of the stance detection procedure is usually from this set: $\{$ Favor, Against, None $\}$. In this tutorial, we will define the core concepts and research problems related to stance detection, present historical and contemporary approaches to stance detection, provide pointers to related resources (datasets and tools), and we will cover outstanding issues and application areas of stance detection. As solutions to stance detection can contribute to significant tasks including trend analysis, opinion surveys, user reviews, personalization, and predictions for referendums and elections, it will continue to stand as an important research problem, mostly on textual content currently, and particularly on social media. Finally, we believe that image and video content will commonly be the subject of stance detection research soon.
\end{abstract}

\section{CCS CONCEPTS}

- Computing methodologies $\rightarrow$ Natural language processing; Machine learning; Language resources; - Information systems $\rightarrow$ Information retrieval; Web and social media search.

\section{KEYWORDS}

Stance detection; Social media analysis; Deep learning; Data streams

\section{ACM Reference Format:}

Dilek Küçük and Fazli Can. 2021. Stance Detection: Concepts, Approaches, Resources, and Outstanding Issues. In Proceedings of the 44th International ACM SIGIR Conference on Research and Development in Information Retrieval (SIGIR '21), July 11-15, 2021, Virtual Event, Canada. ACM, New York, NY, USA, 4 pages. https://doi.org/10.1145/3404835.3462815

ACM acknowledges that this contribution was authored or co-authored by an employee, contractor or affiliate of a national government. As such, the Government retains a nonexclusive, royalty-free right to publish or reproduce this article, or to allow others to do so, for Government purposes only.

SIGIR '21, July 11-15, 2021, Virtual Event, Canada

(C) 2021 Association for Computing Machinery.

ACM ISBN 978-1-4503-8037-9/21/07 . \$ \$15.00

https://doi.org/10.1145/3404835.3462815

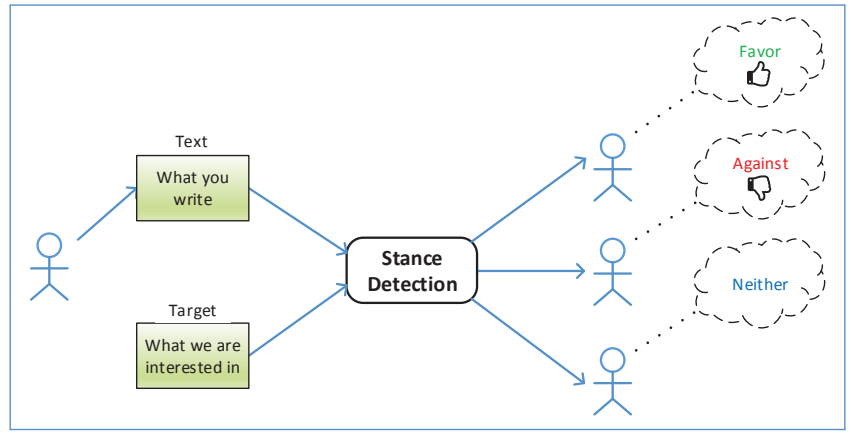

Figure 1: Typical stance detection procedure [19].

\section{INTRODUCTION}

Stance detection has recently emerged as an important research problem in natural language processing (NLP), social media analysis, and information retrieval (IR), as revealed in the number of related papers published particularly since 2015 [19]. It is a research topic considered closely related to sentiment analysis [12] and is commonly defined as the detection of the stance (as Favor, Against, or None) of the text producer towards a target [26, 27]. A visual representation of the stance detection procedure is given in Figure 1 for illustrative purposes, as excerpted from [19].

Apart from sentiment analysis, there are other research problems closely related to stance detection, including emotion detection [30], sarcasm detection [15], irony detection [43], controversy detection [10], and argument mining [23], among others. These related research problems in addition to subproblems of stance detection are demonstrated in Figure 2, as excerpted from [19].

Stance detection is known to have several application areas such as prediction of election/referendum results [21], information retrieval [32], rumour classification [56], and fake news detection ${ }^{1}$ [22]. Particularly based on the latter two application areas, two subproblems of stance detection have emerged, namely, rumour stance detection and fake news stance detection [19]. Definitions of these subproblems along others (multi-target stance detection [33] and cross-target stance detection $[45,48,50])$ will be provided within the course of this tutorial.

An important contribution to the stance detection research is a series of shared tasks on stance detection between 2016 and 2017: an initial competition on English tweets [26], another on Chinese microblog posts [49], and another related competition on Spanish

${ }^{1}$ http://www.fakenewschallenge.org/ 


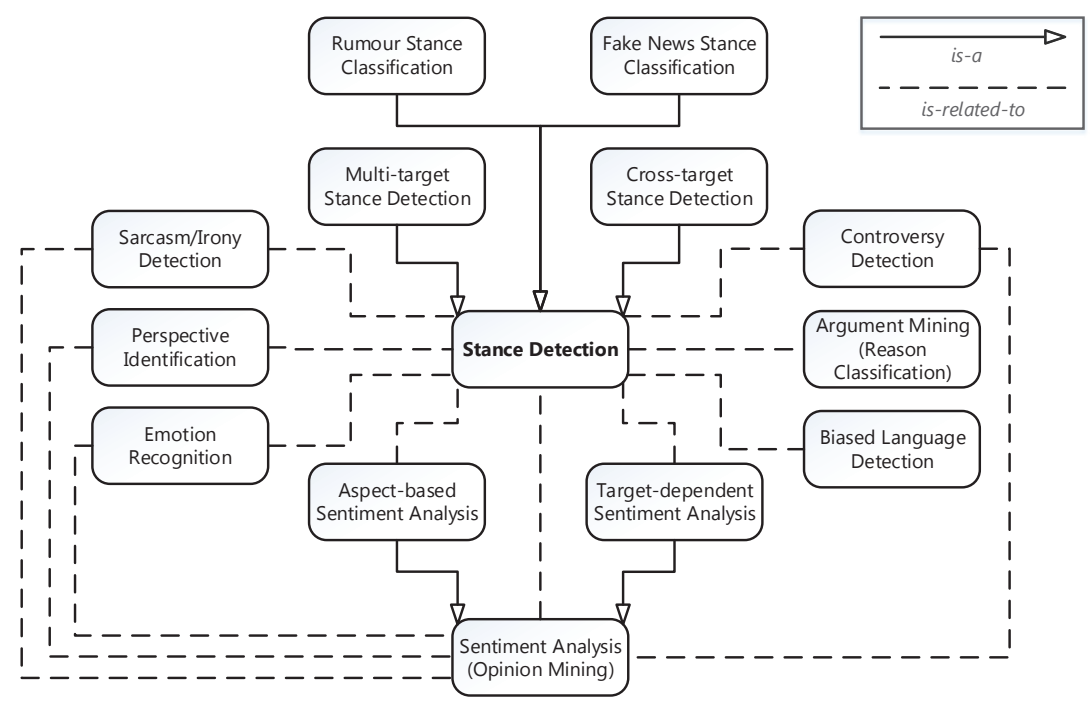

Figure 2: Stance detection and related problems [19].

and Catalan tweets [38]. More recently, a stance detection shared task has also been performed for tweets in Italian [8]. In addition to the evaluation of different approaches for stance detection on microblogs in different languages, these competitions have facilitated the compilation significant annotated datasets [8, 25, 26, 49] for stance detection.

Earlier work on stance detection utilized different rule-based and machine learning based algorithms on a variety of text genres including congressional-floor debates, online debate forums, student essays, and tweets $[4,11,13,29,39]$. Related studies seem to have increased noticeably after the aforementioned initial shared task on stance detection in English tweets [26]. Hence, particularly considering those studies after (and including) this shared task; traditional machine learning approaches like SVM [1, 16, 17, 26, 27, 32, 47] and logistic regression [8, 28, 51, 52], deep learning approaches like LSTM [2, 9, 35, 36, 44] and CNN [42, 46, 53], and ensemble methods $[24,37,40]$ have all been employed for the task of stance detection. Though machine learning approaches like SVM are the most commonly utilized ones up until 2019 , more recent studies tend to apply deep learning algorithms, similar to the case of many tasks in NLP and IR [3, 19].

There also exist significant research efforts to produce annotated datasets and benchmarks for stance detection [18, 20, 31, 34, 55]. Currently, stance-annotated datasets exist for a number of languages including English [25, 34], Catalan [38], Chinese [49], Czech [14], Italian [21], Spanish [38], and Turkish [18]. Yet, in addition to these language-specific datasets, recent work on the compilation of multilingual stance-annotated datasets [20, 41, 55] (where these datasets include annotated samples in other languages such as German and French) will facilitate related research and thereby will hopefully lead to important findings.

There are several lines of future research after having a firm base knowledge about the stance detection problem. Examples include cross-lingual and multilingual stance detection [19, 20], stance detection in data streams [6,54], stance detection on non-textual content, context-sensitive stance detection [19], and exploring the application opportunities of new methods $[5,7]$.

This tutorial aims to cover the core concepts in stance detection together with related research topics, significant approaches to this problem published so far, relevant datasets and other practical resources, application areas, and finally, outstanding issues not sufficiently addressed in the related literature so far. We believe that this tutorial will be beneficial to interested NLP and IR researchers alike, as well as related practitioners.

\section{OBJECTIVES OF THE TUTORIAL}

The objective of this tutorial is to provide an overall understanding of the stance detection problem, which has significant and widespread application opportunities. Particularly, the tutorial attendees will:

- grasp the main stance detection problem, its subproblems, and also those problems closely related to stance detection,

- learn about the evolution of the stance detection literature, and about common algorithms and approaches to this problem,

- be provided with pointers to related datasets and tools so that they can readily begin conducting stance detection experiments themselves,

- learn about the related application areas as well as open research topics regarding stance detection,

- learn some common and favorable practices employed by stance detection researchers and practitioners.

Considering previous tutorials on this topic, we come across a single tutorial titled "Detection and Characterization of Stance on Social Media" which was carried out within the course of 14th International Conference on Web and Social Media (ICWSM-2020). Below listed are the differences between our tutorial on stance detection and above-mentioned previous tutorial: 
- Previous tutorial focuses on social media only while our tutorial will have a broader coverage including other input genres as in fake news stance detection where news articles are under consideration.

- Being a more recent tutorial on the topic, our tutorial will cover more recent work which is a significant advantage as considerable body of work is published on stance detection each year.

- In our tutorial, we will pay particular attention to the subproblems of stance detection and other closely-related problems, as we believe that each one of these topics are fruitful research topics to pursue for both IR and NLP researchers and practitioners.

- Our tutorial will also cover significant application areas and outstanding issues like stance detection on data streams which are relevant to both IR and NLP.

\section{RELEVANCE TO THE IR COMMUNITY}

Stance detection can be considered as a research problem at its juvenile stage since the earliest study that we come across on the topic dates back to 2006 [39] only. But, as emphasized previously, related shared tasks at conferences such as SemEval-2016 [26] have led to a surge on the number of studies conducted on this topic.

We can claim that stance detection is at the crossroads of social media analysis, IR, and NLP. Therefore, related studies have been commonly published at the related conferences such as SIGIR, COLING, WWW, SemEval, EMLNP, ECIR, and EACL. For instance, stance-related studies published at SIGIR conference series include $[10,44,45]$. Some of the journals where stance detection studies have been published include ACM Computing Surveys, ACM Transactions on Internet Technology, Social Network Analysis and Mining, and Computer Speech \& Language.

Hence, we believe that after attending this half-day tutorial on stance detection, researchers and practitioners of social media analysis, IR, and NLP can learn about the state-of-the-art on stance detection and can readily start to create their own research agenda on one of the open research issues regarding stance detection.

\section{INTENDED AUDIENCE}

This tutorial aims to present the core concepts, approaches, resources, application areas, and further research directions of stance detection. Stance detection is an important research problem and has several application areas related to information retrieval. Therefore, we hope that many related researchers and practitioners of social media analysis, information retrieval, and natural language processing (with introductory knowledge) can benefit from this tutorial. There are not any particular prerequisites for the attendees. The presentation files of the tutorial and a list of the relevant bibliography will be provided to the attendees in electronic formats.

\section{TUTORIAL OUTLINE}

Below provided is the outline of our stance detection tutorial.

(1) Introduction

(2) Core concepts and related problems
- Definitions of stance detection, multi-target stance detection, cross-target stance detection, rumour stance classification, and fake news stance detection.

- Interrelationships with related problems: sentiment analysis, emotion recognition, sarcasm/irony detection, controversy detection, and argument mining

(3) Stance detection competitions (shared tasks)

- SemEval-2016 Task 6: Detecting Stance in Tweets

- Shared Task of Stance Detection in Chinese Microblogs at NLPCC-ICCPOL-2016

- Shared Task of Stance Detection in Spanish and Catalan Tweets at IberEval-2017

- SardiStance: Stance Detection Task in Italian Tweets at EVALITA-2020

(4) Historical and contemporary approaches

- Earlier studies on stance detection

- Machine learning based approaches

- Deep learning based approaches

- Ensemble learning approaches

(5) Resources (datasets \& tools)

- Common datasets for stance detection

- Learning tools used and system implementations

(6) Application areas

- Opinion surveys/polling

- Public health surveillance

- Information retrieval

- Stance summarization

- Rumour classification

- Fake news detection

(7) Outstanding issues with an emphasis on data streams

- Cross-lingual and multilingual stance detection

- Stance detection on non-textual data and robots

- Context-sensitive stance detection

- Stance detection in data streams

(8) Concluding remarks

\section{REFERENCES}

[1] Aseel Addawood, Jodi Schneider, and Masooda Bashir. 2017. Stance classification of Twitter debates: the encryption debate as a use case. In Proceedings of the 8th International Conference on Social Media \& Society. 2.

[2] Rabab Alkhalifa and Arkaitz Zubiaga. 2020. QMUL-SDS at SardiStance2020: Leveraging network interactions to boost performance on stance detection using knowledge graphs. arXiv preprint arXiv:2011.01181 (2020).

[3] Emily Allaway, Malavika Srikanth, and Kathleen McKeown. 2021. Adversarial learning for zero-shot stance detection on social media. In Proceedings of the Conference of the North American Chapter of the Association for Computational Linguistics.

[4] Pranav Anand, Marilyn Walker, Rob Abbott, Jean E Fox Tree, Robeson Bowmani, and Michael Minor. 2011. Cats rule and dogs drool!: classifying stance in online debate. In Proceedings of the 2nd Workshop on Computational Approaches to Subjectivity and Sentiment Analysis. 1-9.

[5] Hamed Bonab and Fazli Can. 2019. Less is more: a comprehensive framework for the number of components of ensemble classifiers. IEEE Transactions on Neural Networks and Learning Systems 30, 9 (2019), 2735-2745.

[6] Hamed R Bonab and Fazli Can. 2018. GOOWE: geometrically optimum and online-weighted ensemble classifier for evolving data streams. ACM Transactions on Knowledge Discovery from Data (TKDD) 12, 2 (2018), 25.

[7] Umit Can and Bilal Alatas. 2021. A novel approach for efficient stance detection in online social networks with metaheuristic optimization. Technology in Society 64 (2021), 101501.

[8] Alessandra Teresa Cignarella, Mirko Lai, Cristina Bosco, Viviana Patti, Rosso Paolo, et al. 2020. SardiStance@EVALITA2020: Overview of the task on stance detection in Italian tweets. In EVALITA 2020 Seventh Evaluation Campaign of Natural Language Processing and Speech Tools for Italian. 1-10. 
[9] Kuntal Dey, Ritvik Shrivastava, and Saroj Kaushik. 2018. Topical stance detection for Twitter: A two-phase LSTM model using attention. In European Conference on Information Retrieval. 529-536.

[10] Shiri Dori-Hacohen. 2015. Controversy detection and stance analysis. In Proceedings of the International ACM SIGIR Conference on Research \& Development in Information Retrieval. 1057.

[11] Adam Faulkner. 2014. Automated classification of stance in student essays: an approach using stance target information and the Wikipedia link-based measure. In Proceedings of the International Florida Artificial Intelligence Research Society Conference. 174-179.

[12] Ronen Feldman. 2013. Techniques and applications for sentiment analysis. Commun. ACM 56, 4 (2013), 82-89.

[13] Kazi Saidul Hasan and Vincent Ng. 2013. Stance classification of ideological debates: data, models, features, and constraints. In Proceedings of the International Joint Conference on Natural Language Processing. 1348-1356.

[14] Tomáš Hercig, Peter Krejzl, Barbora Hourová, Josef Steinberger, and Ladislav Lenc. 2017. Detecting stance in Czech news commentaries. In Proceedings of the Conference on Theory and Practice of Information Technologies (ITAT).

[15] Aditya Joshi, Pushpak Bhattacharyya, and Mark J Carman. 2017. Automatic sarcasm detection: a survey. ACM Computing Surveys (CSUR) 50, 5 (2017), 73.

[16] Dilek Küçük. 2017. Stance detection in Turkish tweets. In Proceedings of the International Workshop on Social Media World Sensors.

[17] Dilek Küçük and Fazli Can. 2018. Stance detection on tweets: an SVM-based approach. arXiv preprint arXiv:1803.08910 (2018).

[18] Dilek Küçük and Fazli Can. 2019. A tweet dataset annotated for named entity recognition and stance detection. arXiv preprint arXiv:1901.04787 (2019).

[19] Dilek Küçük and Fazli Can. 2020. Stance detection: A survey. ACM Computing Surveys (CSUR) 53, 1 (2020), 1-37. https://doi.org/10.1145/3369026

[20] Mirko Lai, Alessandra Teresa Cignarella, Delia Irazú Hernández Farías, Cristina Bosco, Viviana Patti, and Paolo Rosso. 2020. Multilingual stance detection in social media political debates. Computer Speech \& Language (2020), 101075.

[21] Mirko Lai, Viviana Patti, Giancarlo Ruffo, and Paolo Rosso. 2018. Stance evolution and Twitter interactions in an Italian political debate. In Proceedings of 23rd International Conference on Natural Language and Information Systems.

[22] David MJ Lazer, Matthew A Baum, Yochai Benkler, Adam J Berinsky, Kelly M Greenhill, Filippo Menczer, Miriam J Metzger, Brendan Nyhan, Gordon Pennycook, David Rothschild, et al. 2018. The science of fake news. Science 359, 6380 (2018), 1094-1096.

[23] Marco Lippi and Paolo Torroni. 2016. Argumentation mining: state of the art and emerging trends. ACM Transactions on Internet Technology (TOIT) 16, 2 (2016), 10.

[24] Can Liu, Wen Li, Bradford Demarest, Yue Chen, Sara Couture, Daniel Dakota, Nikita Haduong, Noah Kaufman, Andrew Lamont, Manan Pancholi, Kenneth Steimel, and Sandra Kübler. 2016. IUCL at SemEval-2016 task 6: an ensemble model for stance detection in Twitter. In Proceedings of the 10th International Workshop on Semantic Evaluation (SemEval-2016). 394-400.

[25] Saif M Mohammad, Svetlana Kiritchenko, Parinaz Sobhani, Xiaodan Zhu, and Colin Cherry. 2016. A dataset for detecting stance in tweets. In Proceedings of the Language Resources and Evaluation Conference. 3945-3952.

[26] Saif M Mohammad, Svetlana Kiritchenko, Parinaz Sobhani, Xiaodan Zhu, and Colin Cherry. 2016. SemEval-2016 task 6: detecting stance in tweets. In Proceedings of the 10th International Workshop on Semantic Evaluation (SemEval-2016). $31-41$.

[27] Saif M Mohammad, Parinaz Sobhani, and Svetlana Kiritchenko. 2017. Stance and sentiment in tweets. ACM Transactions on Internet Technology 17, 3 (2017), Article 26.

[28] Mauridhi Hery Purnomo, Surya Sumpeno, Esther Irawati Setiawan, and Diana Purwitasari. 2017. Biomedical engineering research in the social network analysis era: stance classification for analysis of hoax medical news in social media. Procedia Computer Science 116 (2017), 3-9.

[29] Ashwin Rajadesingan and Huan Liu. 2014. Identifying users with opposing opinions in Twitter debates. In Proceedings of the International Conference on Social Computing, Behavioral-Cultural Modeling, and Prediction. 153-160.

[30] Kashfia Sailunaz, Manmeet Dhaliwal, Jon Rokne, and Reda Alhajj. 2018. Emotion detection from text and speech: a survey. Social Network Analysis and Mining 8, 1 (2018), 28.

[31] Benjamin Schiller, Johannes Daxenberger, and Iryna Gurevych. 2021. Stance detection benchmark: How robust is your stance detection? KI-Künstliche Intelligenz (2021), 1-13.

[32] Anirban Sen, Manjira Sinha, Sandya Mannarswamy, and Shourya Roy. 2018. Stance classification of multi-perspective consumer health information. In Proceedings of the ACM India foint International Conference on Data Science and Management of Data. 273-281.

[33] Parinaz Sobhani. 2017. Stance detection and analysis in social media. Ph.D. Dissertation. Université d'Ottawa/University of Ottawa.

[34] Parinaz Sobhani, Diana Inkpen, and Xiaodan Zhu. 2017. A dataset for multitarget stance detection. In Proceedings of the Conference of the European Chapter of the Association for Computational Linguistics. 551-557.
[35] Qingying Sun, Zhongqing Wang, Shoushan Li, Qiaoming Zhu, and Guodong Zhou. 2018. Stance detection via sentiment information and neural network model. Frontiers of Computer Science (2018).

[36] Oingying Sun, Zhongqing Wang, Oiaoming Zhu, and Guodong Zhou. 2018. Stance detection with hierarchical attention network. In Proceedings of the International Conference on Computational Linguistics. 2399-2409.

[37] Sahil Swami, Ankush Khandelwal, Vinay Singh, Syed Sarfaraz Akhtar, and Manish Shrivastava. 2018. An English-Hindi code-mixed corpus: stance annotation and baseline system. arXiv preprint arXiv:1805.11868 (2018).

[38] Mariona Taulé, M Antonia Martí, Francisco Rangel, Paolo Rosso, Cristina Bosco, and Viviana Patti. 2017. Overview of the task on stance and gender detection in tweets on Catalan independence at IberEval 2017. In Proceedings of the Second Workshop on Evaluation of Human Language Technologies for Iberian Languages (IberEval 2017).

[39] Matt Thomas, Bo Pang, and Lillian Lee. 2006. Get out the vote: determining support or opposition from congressional floor-debate transcripts. In Proceedings of the Conference on Empirical Methods in Natural Language Processing. 327-335.

[40] Martin Tutek, Ivan Sekulic, Paula Gombar, Ivan Paljak, Filip Culinovic, Filip Boltuzic, Mladen Karan, Domagoj Alagić, and Jan Šnajder. 2016. Takelab at SemEval-2016 task 6: stance classification in tweets using a genetic algorithm based ensemble. In Proceedings of the 10th International Workshop on Semantic Evaluation (SemEval-2016). 464-468.

[41] Jannis Vamvas and Rico Sennrich. 2020. X-stance: A multilingual multi-target dataset for stance detection. arXiv preprint arXiv:2003.08385 (2020).

[42] Prashanth Vijayaraghavan, Ivan Sysoev, Soroush Vosoughi, and Deb Roy. 2016. DeepStance at SemEval-2016 task 6: detecting stance in tweets using character and word-level CNNs. In Proceedings of the 10th International Workshop on Semantic Evaluation (SemEval-2016).

[43] Byron C Wallace. 2015. Computational irony: a survey and new perspectives. Artificial Intelligence Review 43, 4 (2015), 467-483.

[44] Penghui Wei, Junjie Lin, and Wenji Mao. 2018. Multi-target stance detection via a dynamic memory-augmented network. In Proceedings of the International ACM SIGIR Conference on Research \& Development in Information Retrieval. 12291232.

[45] Penghui Wei and Wenji Mao. 2019. Modeling transferable topics for cross-target stance detection. In Proceedings of the 42nd International ACM SIGIR Conference on Research and Development in Information Retrieval. 1173-1176.

[46] Wan Wei, Xiao Zhang, Xuqin Liu, Wei Chen, and Tengjiao Wang. 2016. pkudblab at SemEval-2016 task 6: a specific convolutional neural network system for effective stance detection. In Proceedings of the 10th International Workshop on Semantic Evaluation (SemEval-2016). 384-388.

[47] Michael Wojatzki and Torsten Zesch. 2016. Itl.uni-due at SemEval-2016 task 6: stance detection in social media using stacked classifiers. In Proceedings of the 10th International Workshop on Semantic Evaluation (SemEval-2016). 428-433.

[48] Chang Xu, Cecile Paris, Surya Nepal, and Ross Sparks. 2018. Cross-target stance classification with self-attention networks. arXiv preprint arXiv:1805.06593 (2018).

[49] Ruifeng Xu, Yu Zhou, Dongyin Wu, Lin Gui, Jiachen Du, and Yun Xue. 2016. Overview of NLPCC shared task 4: stance detection in Chinese microblogs. In Natural Language Understanding and Intelligent Applications. 907-916.

[50] Bowen Zhang, Min Yang, Xutao Li, Yunming Ye, Xiaofei Xu, and Kuai Dai. 2020. Enhancing cross-target stance detection with transferable semantic-emotion knowledge. In Proceedings of the 58th Annual Meeting of the Association for Computational Linguistics. 3188-3197.

[51] Shaodian Zhang, Lin Qiu, Frank Chen, Weinan Zhang, Yong Yu, and Noémie Elhadad. 2017. We make choices we think are going to save us: debate and stance identification for online breast cancer CAM discussions. In Proceedings of the International Conference on World Wide Web Companion. 1073-1081.

[52] Zhihua Zhang and Man Lan. 2016. ECNU at SemEval 2016 task 6: relevant or not? supportive or not? a two-step learning system for automatic detecting stance in tweets. In Proceedings of the 10th International Workshop on Semantic Evaluation (SemEval-2016). 451-457.

[53] Yiwei Zhou, Alexandra I Cristea, and Lei Shi. 2017. Connecting targets to tweets: semantic attention-based model for target-specific stance detection. In Proceedings of the International Conference on Web Information Systems Engineering. 1832.

[54] Lixing Zhu, Yulan He, and Deyu Zhou. 2020. Neural opinion dynamics model for the prediction of user-level stance dynamics. Information Processing \& Management 57, 2 (2020), 102031.

[55] Elena Zotova, Rodrigo Agerri, and German Rigau. 2021. Semi-automatic generation of multilingual datasets for stance detection in Twitter. Expert Systems with Applications 170 (2021), 114547.

[56] Arkaitz Zubiaga, Ahmet Aker, Kalina Bontcheva, Maria Liakata, and Rob Procter. 2018. Detection and resolution of rumours in social media: a survey. $A C M$ Computing Surveys (CSUR) 51, 2 (2018), 32. 